\title{
Schultheater und Darstellendes Spiel - in Österreich
}

\author{
Dagmar Höfferer-Brunthaler
}

\begin{abstract}
Der nachstehende Text ist ein Beitrag zum neuen Projekt der UCC "Scenario Correspondents". In mehreren Aufsätzen soll der aktuelle Stand der Drama- / Theaterpädagogik in einzelnen Ländern festgehalten werden. Konkret versteht sich dieser Text als ein erster Überblick über den aktuellen Stand der Institutionen rund um den Theaterunterricht in österreichischen Schulen. Er könnte auch als Startschuss für das gesamte Projekt gesehen werden. Es ist geplant, diesem Überblick weitere Informationen aus Österreich folgen zu lassen. Möglicherweise können Abschlussarbeiten (MasterThesen) zu diesem Thema verfasst werden. Diese Zusammenfassung betrachtet die Möglichkeiten fürösterreichischen Lehrer_innen, sich Unterstützung und Hilfe in der Umsetzung theatralen Unterrichts zu holen. Das meint einerseits den Bereich Aus- und Fortbildung, andererseits die konkrete Unterstützung durch Beratung und Finanzierung. Genannt werden auch überregionale Initiativen, Gemeinschaften sowie Kinder- und Jugendfestivals, die allesamt Vernetzung und Kooperation ermöglichen und fördern.
\end{abstract}

\section{Zum Stand der Drama- / Theaterpädagogik in Österreichs Schulen}

Österreich hat eine Zahlvon Vermittlungsprojekten, die Schulen, Schüler_innen und Lehrer_innen und Theaterinstitutionen bzw. Künstler_innen miteinander in Kontakt bringen, Projektunterstützung in verschiedenster Weise anbieten und Plattformen für Dokumentation und Präsentationen anbieten. Sie fördern damit kulturelle Bildung in einem beträchtlichen Ausmaß.

Dieser Text ist eine überarbeitete Version eines Ausschnittes aus einer MasterThesis, die sich mit den derzeitigen Möglichkeiten des Theaterunterrichts an Österreichs Schulen beschäftigt. 


\subsection{Vermittlungsprojekte des Bildungsministeriums}

Allen voran ist KulturKontaktAustria (KKA) (www.kulturkontakt.or.at/) zu nennen, eine Institution, die es bereits seit 1989 gibt. KKA unterstützt sowohl Künstlerinnen und Künstler in ihrer (pädagogisch orientierten und kunstvermittelnden) Tätigkeit und bestärkt Lehrer_innen, kulturelle Bildung anzubieten. Große Projekte, das Schulkulturbudget und einzelne Dialogveranstaltungen machen es Lehrer_innen leicht, mit Künstler_innen zusammen zu arbeiten. Diese Finanzierung ist oft überlebenswichtig für künstlerisch-kulturelles Arbeiten im schulischen Kontext. Kulturkontakt ist direkt dem Bundesministerium für Bildung (BMB) unterstellt und wird vom Bundeskanzleramt (BKA) unterstützt.

KulturKontaktAustria definiert sich selbst als „ein europäisches Kompetenzund Ressourcenzentrum mit den Kernbereichen Kulturvermittlung mit Schulen in Österreich, internationale Bildungskooperation und dem Artists-inResidence-Programm für KünstlerInnen aus dem Ausland“ (KulturKontakt Austria / Über uns).

DieInitiative derBMB Macht | Schule | Theater (www. macht schuletheater. at/) brachte seit 2007 jeweils ein Theater und ein bis zwei Schulen als Partner zusammen. Die Förderung von Kreativität und Fantasie, von Sozialkompetenz und künstlerischem Ausdruck sowie die Auseinandersetzung und Reflexion kultureller Bildungsinhalte waren erklärte Zielsetzungen. Langjährige Partnerschaften zwischen Theaterbetrieben und Schulen sind hier entstanden. Seit dem Frühsommer 2016 geht die Initiative in CULTURE CONNECTED auf.

CULTURE CONNECTED (www. culture-connected.at/) sieht sich als österreichweite Initiative des $\mathrm{BMB}$, Kooperationsprojekte zwischen Schulen und Kulturpartnern zu unterstützen. Diese Kooperation geht jeweils über zwei Jahres. Konzepte werden eingereicht, nach bestimmten Kriterien ausgewählt und unterstützt. Sämtliche künstlerische Sparten sind vertreten, von Architektur bis Theater. Eine konzeptionell und organisatorisch beratende Funktion hat Kulturkontakt weiterhin inne.

Culture connected möchte allen Kindern und Jugendlichen an österreichischen Schulen eine partizipative Teilnahme an Kunst und Kultur ermöglichen. Dafür öffnet sich Culture Connected allen Kulturinstitutionen, -initiativen und -vereinen. Neue Lernformen und -orte werden besonders unterstützt.

Der Landesschulrat für Vorarlberg betreibt seit mehreren Jahren die Initiative Schultheater-Coaches (http://schuleundtheater.vobs.at/projekte/ schultheatercoach/). „Reisende Schultheaterexperten“ bzw. Theaterpädagogen unterstützen Lehrerinnen und Lehrer in allen Belangen theatralen Unterrichtens und bei Inszenierungen. Man fordert sie über den Landesverband für Amateurtheater an.

Ein weitere intensive Kooperation zwischen Theater und Schule sind Lehrerinnen und Lehrer, die Kunstvermittlung in professionellen Theatern betreiben, also Theaterpädagog_innen, die einen Teil ihrer Lehrverpflichtung in Theaterinstitutionen verbringen und sowohl in den Theatern als auch den Schulen theaterpädagogische Vor- und Nachbereitungen einzelner Inszenierungen anbieten. Theaterpädagog_innen werden derzeit im Schauspielhaus Graz und 
im Volkstheater Wien eingesetzt. Diese Zusammenarbeit zwischen Schule und Theater erfreut sich großer Beliebtheit.

\subsection{Bundesverband für Amateurtheater / Landesverbände}

Der Dachverband ÖBV Theater (Österreichischer Bundesverband für außerberufliches Theater) (www.oebvtheater.at/) bekennt sich zwar in seinem Logo zu Schule|Jugend|Amateur, kann aus mangelnder finanzieller Unterstützung momentan für schulische Theaterarbeit nicht eintreten. Ein Fachbereich Schulund Jugendtheater ist zwar derzeit mit zwei Personen besetzt, aber quasi untätig.

Was die einzelnen Mitgliedsverbände des ÖBVTheater anbieten, sind Aus- und Fortbildung, konkrete Hilfestellungen für Theatergruppen und diverse Publikationen. Diese professionelle Unterstützung können auch schulische Gruppen in Anspruch nehmen, wenn sie Mitglied des jeweiligen Landesverbandes sind.

Die an den ÖBV Theater angegliederten Mitgliedsverbände, die Landesverbände für Amateurtheater können die schulische Theaterarbeit unterschiedlich unterstützen.

- Der Theater Service Kärnten (www.theater-service-kaernten.com/) veranstaltet regelmäßig Kinder- und Jugendtheater-Festivals, Schulen sind als Teilnehmer und als Zuschauer vertreten.

- ATiNOE - Außerberufliches Theater in Niederösterreich (www.atinoe. at/) hat keine eigene Schultheater-Schiene. Schulgruppen können Mitglied werden und kommen in den Genuss der Angebote.

- Im Bundesland Niederösterreich gibt es eine höchst aktive Arbeitsgemeinschaft ARGE Schulspiel gibt (www.schulspiel-noe.at/). Die Koordinatorinnen der einzelnen Bezirke treffen einander regelmäßig. Es gibt Angebote zu Aus- und Fortbildungen, Beratung, eine Stückebörse und einen Referentenpool.

- Der oberösterreichische Verband Amateurtheater Oberösterreich (www . amateurtheater-ooe.at/) bietet keinerlei schulische Aktivitäten. Das Schultheater und das Fach „Darstellendes Spiel“ sind in Oberösterreich stark entwickelt.

- In der Steiermark gibt es Laut! (laut.or.at/), den Landesverband für außerberufliches Theater in der Steiermark. LAUT! kooperiert mit regionalen, nationalen und internationalen Verbänden und Interessenvertretungen. Schultheatergruppen können Mitglieder werden, spezielle schulische Belange werden nicht erfasst. Erfahrungsaustausch, Vernetzung sowie Entwicklung und Umsetzung von Theaterprojekten sind erklärte Ziele.

- Der Theater Verband Tirol (www.theaterverbandtirol.at/) fördert alle Richtungen des Theaters bzw. des darstellenden Spiels in seinem 
Bundesland. Die umfangreiche Homepage weist den Fachbereich „Schultheater \& Dramapädagogik" auf, der aber zur Zeit nicht besetzt ist. Einige Aktivitäten, die den schulischen Kontext betreffen, werden im Bereich „Theaterpädagogik“ abgedeckt.

- DerLandesverband Vorarlberg Amateurtheater (lva-theaterservice. at/ ) ist der einzige Landesverband, der einen aktiven Fachbereich "Schultheater \& Theaterpädagogik“ verzeichnet. Die Theater-Coaches sind lebendiger Ausdruck dafür.

- Der Landesverband für außerberufliches Theater, Darstellendes Spiel und Dramapädagogik in Wien (www.atheaterwien.at/) versucht eine Neukonstituierung. ATHEATER betreibt explizit die Förderung des Darstellenden Spiels in- und außerhalb der Schule - durch Aus- und Fortbildung, Beratung und Unterstützung. ATHEATER versteht sich auch als Ansprechpartner der außerberuflichen Theatergruppen in Wien. Es ist zu hoffen, dass ATHEATER in Zukunft wieder ausreichend Kapazitäten besitzt, theatralen Unterricht in Schulen zu unterstützen.

- Der Salzburger Amateurtheaterverband (www.sav-theater.at/) ist nicht dem ÖBV Theater angegliedert. Auch dieser Theaterverband setzt keine Aktivitäten im schulischen Kontext.

- Im Burgenland existiert seit Jahren kein Verband, der sich mit außerberuflichem Theater beschäftigt. Es können keine schulischen Aktivitäten (Darstellendes Spiel) verzeichnet werden (außer Einzelinitiativen).

\subsection{Vereine und Initiativen}

Assitej Austria (www.assitej.at/ueber/), Österreich-Department der internationalen Vereinigung, stellt die Schnittstelle zwischen Künstlerinnen und Künstlern, Produzierenden, dem Publikum, den Veranstaltern, Theaterpädagog_innen und Kulturpolitiker_innen sowie verschiedenen Medien dar. Man versucht seit Jahrzehnten, die Qualität der professionellen darstellenden Kunst für Kinder und Jugendliche in ganz Österreich zu verbessern. Internationale Produktionen (die während verschiedener Festivals gezeigt werden) beweisen seit Jahren eine enorme Qualität. Eine Zusammenarbeit mit den Lehrenden existiert im Rahmen dieser Festivals. Die Theaterpädagog_innen verstehen sich meist als Theaterschaffende, also mehr als Künstler_innen denn als Pädagog_innen.

IDEA (International Drama/Theater and Education Association) (www. ideadrama.org/) existiert seit 1992. Die Vereinigung setzt sich weltweit für die Förderung von Drama in Education ein. Neben vernetzenden Treffen und gemeinsamen Projekten veranstaltet IDEA internationale Kongresse zu aktuellen inhaltlich im pädagogischen Feld liegenden Themen. Innerhalb der Gemeinschaft wird auch die Forschung von Theorie und Praxis forciert. 
Der Weiterbestand des Vereins und die Fortsetzung seiner Aktivitäten sowie die Ausweitung der internationalen Kontakte liegen in den Händen des ÖBV Theater als Bundesverband und dem Vorstand von IDEA. Austria, der aus engagierten Einzelpersonen besteht. Die Unterstützung durch das Bildungsministerium wurde vor einigen Jahren eingestellt, 2016 konnte eine Beteiligung des BMB am Kongress Drama in Education erreicht werden. IDEA Austria ist bestrebt, die Ausrichtung der Kongresse beizubehalten und sie für Lehrerinnen und Lehrer weiterhin attraktiv zu machen. Auch für die anderen Aktivitäten würde IDEA Austria die Unterstützung durch die Schulbehörden benötigen.

Der Kongress „Drama in Education“ wurde erstmals 1974 abgehalten, anfänglich als internationales Seminar mit hochwertigen Referent_innen, gedacht zur Aus- und Fortbildung für Spieler_innen. Der damalige Geschäftsführer des ÖBV Theater, Alfred Meschnigg, der auch das Schulspiel als Unverbindliche Übung in die Schulen hereinholte, war Pate dieser Idee. Daraus entwickelte sich der Kongress „Drama in Education“, der regelmäßig stattfindet. Nationale und internationale Teilnehmer trafen und treffen auf erstklassige Referent_innen aus der ganzen Welt (z.B. Augusto Boal, Judith Ackroyd, Alan Owens, Marcel Cremer, etc.).

Ab 1992 wurde der Kongress zweijährig veranstaltet, zuerst organisiert von ÖBV Theater in der Burg Schlaining (Burgenland). Derzeit ist der Kongressort das Bildungshaus Schloss Retzhof (Steiermark, bei Leibnitz). Die Ergebnisse (theoretische Grundlagen, keynotes und der methodische Aufbau der praktischen Arbeit) sind in den Dokumentationen nachzulesen. Der nächste Kongress findet von 7.-12. April 2017 statt und steht unter dem Thema „Changes - Challenges - Choices - die Kunst, das Richtige zu tun“.

Unterstützung erfährt IDEA auch durch IATA/AITA asbl (www.aitaiata. org/gil/), einer weltweiten Organisation für Amateur-Theater, deren Teilorganisation IDEA ist. Die Ziele der AITA/IATA sind, Verständnis für theatrales Lernen zu entwickeln und Bildung durch Theater zu ermöglichen.

Formen des Bürgertheaters und Theaterklubs (für alle Altersstufen) sind für die hiesigen professionellen Theatereinrichtungen mittlerweile selbstverständlich geworden, die Kooperation mit Schulen dabei ist unterschiedlich. Das liegt vermutlich auch daran, dass die Theaterpädagog_innen vielfach nicht aus dem schulischen Umfeld kommen, teilweise das Schulsystem Österreichs auch nicht ausreichend kennen. Hier läge Potential für konstruktive, langfristig wirkende Zusammenarbeit. Dabei könnte das Bildungsministerium eine wichtige Rolle spielen.

\subsection{Arbeitsgemeinschaften}

Als österreichweit tätige Arbeitsgemeinschaft für Theater in der Schule BAGTIS (www.bag-tis.at/) beschäftigt sich eine Gruppe engagierter Lehrerinnen und Lehrer aller Schulformen mit der Stärkung und Förderung theatralen Unterrichts. BAGTIS ist Teil des Zentrums für schulische Kulturarbeit und 
untersteht dem BMB.

Das Engagement der Arbeitsgemeinschaft erstreckt sich auf einzelne Initiativen und der Weiterentwicklung theatraler Bildungsinhalte. Eine Implementierung eines eigenen Faches wäre wünschenswert, ist derzeit jedoch nicht in Sicht. Ein Kompetenzkatalog: Katalog Schüler-Innen:Kompetenzen (2015) ist in zweijähriger Arbeit entstanden und bildet eine hervorragende Diskussionsgrundlage zum Kompetenzbegriff. Die Förderung der Theaterarbeit im Elementar- und Grundschulbereich sowie verstärkt in der Lehrer_innen-Bildung wäre ein großes Anliegen. Es gibt regelmäßige Treffen in den Bundesländern und eine jährliche österreichweite Bundestagung.

Im Jahr 2016 organisierte das ZSK (Bundeszentrum für schulische Kulturarbeit) anlässlich der UNESCO International Arts Education Week (23. bis 29. Mai 2016) einen Aktionstag „Kulturelle Bildung in Schulen“. Auch die Lehrer_innen von BAGTIS nahmen daran teil. Es gab etwa 350 Projekte aus verschiedenen künstlerischen Sparten. Eine Gesamtdokumentation befindet sich auf der ZSK-Seite (www . bundeszentrum-zsk. at/aktionstag).

\subsection{Aus- und Fortbildung}

Es existieren in Österreich mehrere Möglichkeiten eine Ausbildung zur Theaterpädagogin/ zum Theaterpädagogen zu absolvieren. Sie sind zeitund kostenintensiv und garantieren derzeit keine Anstellung im Schulbereich. Es gibt Ausbildungsgänge zum Spielleiter, die von Lehrerinnen und Lehrern genutzt werden können.

- Masterlehrgang „Theaterpädagogik“ der KPH Wien/Krems (www . kphvie . ac.at/institute/zentrum-fuer-weiterbildung/lehrgaenge-im-ueberblick. $h t m 1$ )

- Masterlehrgang „Lernen durch Darstellen - Theater-Pädagogik“ der Privaten Pädagogischen Hochschule der Diözese Linz (www . phdl . at/aktuell/ aktuell_detailansicht/article/lernen-durch-darstellen-theaterpaedagogik/)

- Ausbildung in Theater- und Schauspielpädagogik (Institut Angewandtes Theater - IFANT), geteilt in Grundlagen-Lehrgang und Aufbau-Lehrgang (theaterpaedagogik-ausbildung.at/)

- Theaterpädagogischer Lehrgang SSpielen, was ist, verändert die Welt. Theater als Experimentierfeld - Entfaltung persönlicher und sozialer Kompetenz"des Vereines spectACT, Verein für politisches und soziales Theater, in Zusammenarbeit mit dem Haus der Begegnung Innsbruck (www.spectact.at/ausbildung)

Eine langjährige „Theaterinstitution“ sei hier zu erwähnen, die seit mehreren Jahrzehnten Workshops mit nationalen und internationalen Referent_innen 
ausschreibt. Der Zustrom ist nach wie vor ungebrochen: Es sind mehr als 100 Lehrerinnen und Lehrer, die ihre vorletzte Ferienwoche regelmäßig in Hollabrunn (NÖ) verbringen. Die PH Niederösterreich veranstaltet mit Unterstützung durch das jeweilige Bildungsministerium seit nunmehr 28 Jahren diese Fortbildung (Sommerworkshop Darstellendes Spiel). Die Tradition geht auf Initiative von Mag. Karl Eigenbauer und sein Team zurück, der nahezu von Beginn an für dieser Sommerwoche verantwortlich ist.

Die Pädagogischen Hochschulen Österreichs bieten in unterschiedlichem Umfang und Intensität Fortbildungen zum Fach Darstellendes Spiel und verwandten Themen an. Genauere Angaben möge man den aktuellen Vorlesungsverzeichnissen entnehmen. Leider muss festgestellt werden, dass die Theaterworkshops trotz regen Zuspruchs aus budgetären Gründen ausgedünnt werden. In diesem Zusammenhang sei der Beitrag Egon Tureceks in der Zeitschrift Erziehung und Unterricht 3-4 (2015) erwähnt.

Die Zahl der Einzelinitiativen steigt in den letzten Jahren sprunghaft. Sämtliche Aus- und Weiterbildungen sowie Workshops werden meist von Einzelpersonen oder Theatervereinen organisiert und angeboten. Es ist zu vermuten, dass Gewinnorientierung hinter manchen Angeboten steckt; die Qualität ist unterschiedlich. Häufig werden Seminare „zum Einstieg in die Theaterwelt“ beworben. Inwieweit diese Theaterarbeit pädagogisch orientiert ist, wäre im Einzelfall zu prüfen. Viele Referent_innen nennen sich „Theaterpädagog_in“, diese Tätigkeitsbezeichnung ist rechtlich in Österreich ungeschützt.

Eine Veranstaltung, die professionelle Produktionen mit Workshops (für jedermann) verbindet, ist die Internationale Sommerakademie für Theater Graz (http://somak.at/). Sie läuft mittlerweile dreißig Jahre, verbindet klassische Workshops wie Clowntheater, Pantomime und Sprechtechnik mit außergewöhnlichen Angeboten (Bühnenkampf, Audition/Casting-Training oder Luftakrobatik). Die Workshops sind nicht ausschließlich für Pädagog_innen gedacht.

\subsection{Kinder- und Jugendtheater-Festivals}

Theaterfestivals für Kinder- und Jugendliche werden häufig entweder von professionellen Theaterinstitutionen (szene bunte wähne, Schäxpir, Junges Volkstheater, Dschungel, etc.) oder von Amateurgruppen (Jugendtheaterfestivals in Kärnten, Vorarlberg, Wien, Oberösterreich, etc.) bespielt. Der ÖBV Theater bzw. die einzelnen Landesverbände schreiben landes- und österreichweite Festivals aus, manchmal zeigen professionelle Gruppen neben schulischen und außerschulischen Theatergruppen ihre Inszenierungen. Meist präsentieren diese Festivals eine Mischung aus nationalen und internationalen Produktionen. Die menschliche Begegnung der jungen Menschen und der fachliche Austausch sind meist wesentlicher Bestandteil dieser Veranstaltungen.

Insgesamt sind Festivals für Schultheatergruppen aus verschiedenen Gründen rückläufig. Eine Belebung der Festival-Szene wäre wünschenswert, über die Bundesländer hinaus gehend und nach dem Vorbild der Kinder- und 
Jugendtheater-Festivals im deutschsprachigen Raum. Der ÖBV veranstaltet jedes Jahr ein Jugendtheaterfestival; Teilnehmer sind auch schulische Gruppen.

\section{Ausblick}

Alle diese Initiativen (und diejenigen, die hier nicht Eingang gefunden haben), könnten bei entsprechender Bündelung und Koordination theatralen Unterricht („Methode Drama“) als Unterrichtsprinzip und die Einführung von „Theater als Fach“ unterstützen. Die Kompetenzkataloge für die Grundstufe, die Sekundarstufe I und II gäbe es bereits. Derzeit stehen allerdings andere Ziele im Vordergrund.

Es bräuchte eine umfassende Diskussion und eine Gesamtkonzept, wohin sich die pädagogische Theaterarbeit (als ein Teil kultureller Bildung) hin entwickeln soll. Die Verbände für das Außerberufliche Theater können im Amateurtheaterbereich die Arbeit mit Jugendlichen und Kindern abdecken. Der ÖBV Theater und seine Mitgliedsverbände sind aus budgetären Gründen nicht in der Lage, den Schulbereich abzudecken.

Das Ministerium hat in den letzten Jahren die Agenden mehrfach weiter gereicht. Derzeit bemüht sich das ZSK - Zentrum für schulische Kulturarbeit (www.bundeszentrum-zsk.at/) konzentriert darum, die Anliegen aller künstlerischen Fächer zu vertreten. Diskussionen um ästhetische Erziehung in allen Kunstsparten und das Angebot fächerübergreifender Veranstaltungen sind ermutigend, in absehbarer Zeit einzelne theoretische Ansätze praktisch verwirklichen zu können. Eine Zusammenarbeit aller künstlerischen Fächer wäre in diesem Zusammenhang wünschenswert.

Die Lehrerinnen und Lehrer aller dieser Fächer sind eine große Gruppe, ihre Anliegen und Forderungen werden hoffentlich in den nächsten Jahren zunehmend gehört.

\section{Bibliografie}

Höfferer-Brunthaler, Dagmar (2015): Darstellendes Spiel: Sehr gut - Von der Unterrichtsmethode zum Maturafach. Master-Thesis zur Erlangung des akademischen Grades Master of Arts (MA) an der Kirchlich-Pädagogischen Hochschule Wien-Krems

Turecek, Egon (2015): Theaterpädagogische Fort- und Weiterbildungsmöglichkeiten an Pädagogischen Hochschulen in Österreich. In: Erziehung \& Unterricht 165/3-4, 312-314. Schwerpunkt: Theaterpädagogik in der Schule

Arbeitsgemeinschaft ARGE Schulspiel. www. schulspiel-noe.at/ [zuletzt aufgerufen 17.12.2016]

Arbeitsgruppe SchülerInnen: Kompetenzen (ARGE [S:K]) der Bundesarbeitsgemeinschaft Theater in der Schule (BAGTIS) (2015): 
Theater in der Schule Kompetenz: Katalog. SchülerInnen: Kompetenzen. Bundeszentrum für schulische Kulturarbeit (ZSK) im Auftrag des BMBF (Bundesministerium für Bildung und Frauen).

www.bagtis.at/images/PDEs/Kompetenz-Katalog.pdf [zuletzt aufgerufen 17.12.2016] oder

www . bmb.gv . at/schulen/schubf/se/SchuelerInnenkompetenzen_Theater_ in_der_Schule.pdf?515347 [zuletzt aufgerufen 17.12.2016]

Assitej Austria.www .assitej .at/ueber/ [zuletzt aufgerufen 17.12.2016]

ATHEATER - Landesverband für außerberufliches Theater, Darstellendes Spiel und Dramapädagogik in Wien. www . atheaterwien.at/ [zuletzt aufgerufen 17.12.2016]

ATiNOE - Außerberufliches Theater in Niederösterreich. www . atinoe .at/ [zuletzt aufgerufen 17.12.2016]

Bundesarbeitsgemeinschaft für Theater in der Schule - BAGTIS. www. bag-tis.at/ [zuletzt aufgerufen 17.12.2016]

Culture Connected. Kooperation zwischen Schulen und Kulturpartnern. www.culture-connected.at/ [zuletzt aufgerufen 17.12.2016]

IATA/AITA asbl. www . aitaiata.org/gil/ [zuletzt aufgerufen 17.12.2016]

IDEA (International Drama/Theater and Education Association). www. ideadrama.org/ [zuletzt aufgerufen 17.12.2016]

Internationale Sommerakademie für Theater Graz. http://somak . at / [zuletzt aufgerufen 17.12.2016]

KulturKontakt Austria. www.kulturkontakt.or.at/ [zuletzt aufgerufen 17.12.2016]

Landesschulrat für Vorarlberg. Schule und Theater. Projekte Schultheatercoach. schuleundtheater.vobs.at/projekte/schultheatercoach/ [zuletzt aufgerufen 17.12.2016]

Landesverband Vorarlberg Amateurtheater. http://1va-theaterservice.at/ [zuletzt aufgerufen 17.12.2016]

Laut! - Landesverband für außerberufliches Theater in der Steiermark. http://laut.or.at/ [zuletzt aufgerufen 17.12.2016]

Macht | Schule | Theater.www.machtschuletheater.at/ [zuletzt aufgerufen 17.12.2016]

Salzburger Amateurtheaterverband. www. sav-theater.at/ [zuletzt aufgerufen 17.12.2016]

Theater Verband Tirol. www . theaterverbandtirol.at/ [zuletzt aufgerufen 17.12.2016]

TheaterServiceKärnten. Amateurtheaterverband.

www . theater-service-kaernten.com/ [zuletzt aufgerufen 17.12.2016]

Verband Amateurtheater Oberösterreich. www . amateurtheater-ooe . at [zuletzt aufgerufen 17.12.2016] 
ZSK - Zentrum für schulische Kulturarbeit. www .bundes zentrum-zsk .at/, siehe auch http://www. bundeszentrum-zsk.at/aktionstag [zuletzt aufgerufen 17.12.2016]

\section{Internet-Quellen}

Arbeitsgemeinschaft ARGE Schulspiel. www.schulspiel-noe.at/ [zuletzt aufgerufen 17.12.2016]

Arbeitsgruppe SchülerInnen: Kompetenzen (ARGE [S:K]) der Bundesarbeitsgemeinschaft Theater in der Schule (BAGTIS) (2015): Theater in der Schule Kompetenz: Katalog. SchülerInnen: Kompetenzen. Bundeszentrum für schulische Kulturarbeit (ZSK) im Auftrag des BMBF (Bundesministerium für Bildung und Frauen).

Www.bagtis.at/images/PDEs/Kompetenz-Katalog.pdf [zuletzt aufgerufen 17.12.2016] oder

www.bmb.gv .at/schulen/schubf/se/SchuelerInnenkompetenzen_Theater_

in_der_Schule.pdf?515347 [zuletzt aufgerufen 17.12.2016]

Assitej Austria.www.assitej . at/ueber/ [zuletzt aufgerufen 17.12.2016]

ATHEATER - Landesverband für außerberufliches Theater, Darstellendes Spiel und Dramapädagogik in Wien. www . atheaterwien. at/ [zuletzt aufgerufen 17.12.2016]

ATiNOE - Außerberufliches Theater in Niederösterreich. www . atinoe .at/ [zuletzt aufgerufen 17.12.2016]

Bundesarbeitsgemeinschaft für Theater in der Schule - BAGTIS. Www .bag-tis.at/ [zuletzt aufgerufen 17.12.2016]

Culture Connected. Kooperation zwischen Schulen und Kulturpartnern. www.culture-connected.at/ [zuletzt aufgerufen 17.12.2016]

IATA/AITA asbl. www. aitaiata.org/gil/ [zuletzt aufgerufen 17.12.2016]

IDEA (International Drama/Theater and Education Association). www. ideadrama.org/ [zuletzt aufgerufen 17.12.2016]

Internationale Sommerakademie für Theater Graz. http://somak .at / [zuletzt aufgerufen 17.12.2016]

KulturKontakt Austria. www.kulturkontakt.or.at/ [zuletzt aufgerufen 17.12.2016]

Landesschulrat für Vorarlberg. Schule und Theater. Projekte Schultheatercoach.

schuleundtheater.vobs.at/projekte/schultheatercoach/ [zuletzt aufgerufen 17.12.2016]

Landesverband Vorarlberg Amateurtheater. http://1va-theaterservice.at/ [zuletzt aufgerufen 17.12.2016]

Laut! - Landesverband für außerberufliches Theater in der Steiermark. http://laut.or.at/ [zuletzt aufgerufen 17.12.2016]

Macht | Schule | Theater. www .machtschuletheater.at/ [zuletzt aufgerufen 17.12.2016] 
Salzburger Amateurtheaterverband. www.sav-theater.at/ [zuletzt aufgerufen 17.12.2016]

Theater Verband Tirol. www .theaterverbandtirol.at/ [zuletzt aufgerufen 17.12.2016]

TheaterServiceKärnten. Amateurtheaterverband.

www.theater-service-kaernten.com/ [zuletzt aufgerufen 17.12.2016]

Verband Amateurtheater Oberösterreich. www . amateurtheater-ooe.at [zuletzt aufgerufen 17.12.2016]

ZSK - Zentrum für schulische Kulturarbeit. www . bundes zentrum-zsk .at/, siehe auch http://www. bundeszentrum-zsk. at/aktionstag [zuletzt aufgerufen 17.12.2016] 